\title{
Structural and transport properties of aluminum atomic wires
}

\author{
Gianni Taraschi \\ Centre for the Physics of Materials and Department of Physics, McGill University, Montreal, Quebec, Canada H3A 2T8 \\ José-Luis Mozos \\ Laboratory of Physics, Helsinki University of Technology, FIN-02150 Espoo, Finland \\ C. C. Wan and Hong Guo \\ Centre for the Physics of Materials and Department of Physics, McGill University, Montreal, Quebec, Canada H3A 2T8
}

Jian Wang

Department of Physics, The University of Hong Kong, Pokfulam Road, Hong Kong

(Received 2 October 1997; revised manuscript received 29 July 1998)

\begin{abstract}
We report a first-principles calculation of structural properties and quantum conductance of aluminum atomic wires. Our data together with a simple model allows us to predict the behavior of the elastic constant $C_{11}$ as a function of the cross-sectional size of the free-standing wires. The quantum molecular dynamics, performed at both 0 and $300 \mathrm{~K}$, provides information concerning the stability of these atomic wires. For the most stable wire, relaxation at $0 \mathrm{~K}$ causes a change of approximately $2-4 \%$ in atomic positions, and room temperature contributes another $4-6 \%$. We obtain the quantum conductance of these wires by combining density functional theory and a three-dimensional evaluation of the scattering matrix. The structures obtained from the quantum molecular-dynamics simulations are examined and transport properties compared. [S0163-1829(98)01444-1]
\end{abstract}

\section{INTRODUCTION}

Free-standing atomic wires, with cross sections as small as a few square nanometers, can now be fabricated using several recently developed experimental techniques. ${ }^{1-6}$ Among the many exciting discoveries on these atomic-scale objects is the observed conductance quantization at elevated temperatures including room temperature. These atomicsized nanostructures promise to have a useful impact on electronic applications. In principle, nanostructure devices can have high operation speeds, low power dissipation, and high packing density. These attributes make them attractive from a technological point of view; however, there are still many obstacles that need to be resolved. In particular, fabricability of atomic-scale nanostructures can be a difficult task due the lack of control over individual atoms. So far many groups have reported successful fabrication of nanowires with various sizes, using a variety of different methods, including nanolithography, ${ }^{1,3}$ scanning tunneling microscopy (STM) and atomic force microscopy, ${ }^{6}$ molecular-beam epitaxy, and porous material templates. ${ }^{2}$ Nanowires exhibiting unusual optoelectronic and electronic properties have been reported, including a direct band gap for porous Si nanowires and conductance quantization. These properties are very useful technologically, but some of them are sensitive to structural imperfections due to both atomic relaxation and temperatureinduced vibrations. Hence it is important to know the amount of disorder introduced by relaxation and temperature, and their influence on the conductance quantization of nanowires. It is also very interesting to understand the crossover of various mechanical properties from nanoscale to macroscale ${ }^{7}$ sizes. principles theoretical methods, the mechanic and quantum transport properties of atomic wires schematically shown in Fig. 1. The wires consist of an atomic section connecting to two long leads. In this work we shall examine aluminum atomic wires. Charge carriers enter the wire from a lead, scatter by the atomic junction, and exit to the second lead or

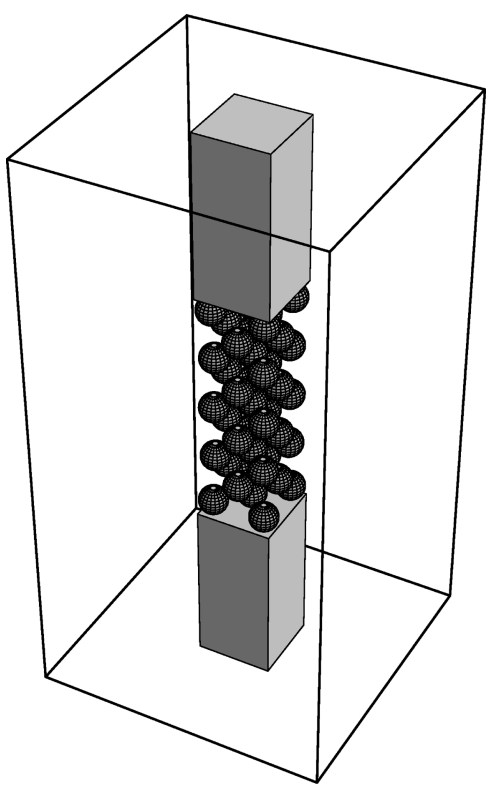

FIG. 1. Schematic plot of a long quantum wire where there is an atomic section and two 3D jellium leads. The whole system is included into a supercell for $a b$ initio total-energy calculations. The atomic positions are determined by quantum molecular dynamics. 
reflect back. For cases with a single Xe atom sandwiched in between two planar jellium electrodes, Ref. 8 reported quantitative agreement between the first-principles prediction and experimental measurements. For the large wires studied here the theoretical calculation becomes much more extended. A complete investigation thus involves two related aspects: (1) the determination of atomic positions and the study of mechanic properties; (2) the prediction of quantum conductance of the wires. This is a very difficult task because any firstprinciples study must include the atomic degrees of freedom: the usual continuum approach using the effective mass approximation familiar in mesoscopic physics cannot give the desired quantitative answer to the quantum conductance of these atomic wires. Our investigation combines the firstprinciple quantum molecular dynamics with the solution of a three-dimensional (3D) quantum scattering problem, thus we are able to make quantitative predictions to these two aspects.

On the mechanical properties of the atomic wires, we investigate the structural stability and certain elastic properties on the atomic scale. We emphasis the small size of the wire, and we answer a number of very relevant questions from a first-principles calculation: what is the crossover behavior of the elastic constant $C_{11}$ as the cross section of the atomic wire is increased? How large are the changes of atomic positions due to relaxation and finite temperature? Can we rationalize our results, which are based on microscopic calculations, using an effective continuum model? These are very general questions concerning important properties of atomicwire fabrication. The microscopic information can only be reliably calculated using ab initio methods and this will be our approach.

On the quantum transport properties of the atomic wires, we investigate the property and quality of conductance quantization. There are a number of investigations on transport through atomic scale objects, especially on the atomic arrangement of a tip near a substrate such as that of the scanning tunneling microscope. ${ }^{6,8-17}$ Transport calculations have also been performed for organic molecules ${ }^{18,19}$ and carbon nanotubes. ${ }^{20}$ A first-principles quantum scattering approach including the atomic degree of freedom has been applied to a chain of atoms sandwiched in between planar electrodes, ${ }^{8}$ or connected to three-dimensional (3D) leads. ${ }^{21,22}$ Since fabrication of free-standing atomic wires becomes increasingly routine, there is a clear need to theoretically predict the quantum transport properties of atomic scale wires and systems beyond the few-atom systems studied so far. Our transport calculations to be reported below examine wires consisting 39 and 41 atoms in their atomic section with the structure determined at various temperatures.

The rest of the paper is organized as follows. In the next section we present mechanical properties of the atomic wires as determined by quantum molecular dynamics. Section III presents the quantum conductance of these wires. Section IV gives the summary.

\section{STRUCTURAL PROPERTIES}

The atomic wires considered in our quantum moleculardynamics (QMD) simulations were a simple aluminum chain, a $1 \times 1 \mathrm{fcc}$ wire without corner atoms, a $1 \times 1$ and 2

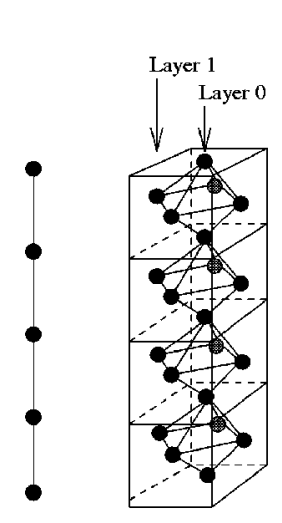

(a)

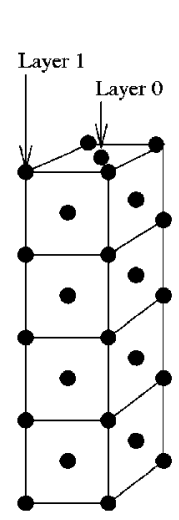

(c)

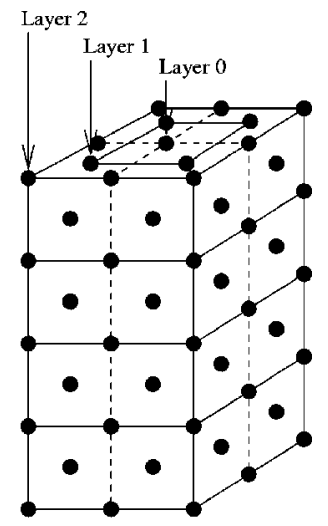

(d)
FIG. 2. Al nanowires studied: (a) Al chain (5 atoms), (b) $1 \times 1$ fcc wire without corner atoms (39 atoms), (c) $1 \times 1 \mathrm{fcc}$ wire (41 atoms), (d) $2 \times 2$ fcc wire (113 atoms).

$\times 2 \mathrm{fcc}$ wire, as shown in Fig. 2. We also performed static total-energy calculations to determine the elastic constant $C_{11}$ along the length of the wire for the $1 \times 1,2 \times 2$, and 3 $\times 3$ nanowires. For these static calculations, the wires considered were infinite, and the supercell length along the wire was chosen to be the lattice constant; $39 k$-sampling points were used along the length of the wire.

To conduct the first-principles calculations we used an approach similar to that of Sankey and Niklewski. ${ }^{23}$ The technique uses the Harris approximation, ${ }^{24}$ the local-density approximation, the pseudopotential of Ref. 25, a minimal $s p^{3}$ atomic orbital basis set, and diagonalization to solve the Kohn-Sham equations. In addition, the atomic orbitals are confined within a radius of $r_{c}$, so that they vanish precisely beyond this distance. This reduces the numerical effort since many of the overlap and Hamiltonian matrix elements for distant atoms are exactly zero. The other nonzero matrix elements are evaluated using pretabulated integral tables, which only need to be calculated once. Although the method is not as accurate as plane-wave techniques, it makes up for this in speed and flexibility. This technique has been extensively tested on many systems including clusters, surfaces, and bulk ${ }^{26-30}$ and is quite suitable for our purposes. After evaluating the forces on the atoms from this ab initio technique, we obtain the structural energy minima by numerically integrating the damped classical equations of motion $\mathbf{F}=m d^{2} \mathbf{r} / d t^{2}+m \gamma d \mathbf{r} / d t$, where $\gamma$ is the damping coefficient. Finite-temperature simulations were performed using Langevin dynamics, ${ }^{31}$ in which a temperature-dependent noise term is added to the classical equations of motion. Finally, in all our nanowire calculations, the $\mathrm{Al}$ atomic orbital confining radius was $r_{c}=6$ a.u., and the QMD time step was 3 fs.

Our results can be grouped into static and dynamic categories.

\section{A. Static}

On the static calculations, we conducted total energy scaling evaluations of the elastic constant $C_{11}$ along the length of the nanowires. In particular, we calculated the total energy per unit volume $E / V$ as a function of the strain $e_{11}=(a$ $\left.-a_{o}\right) / a_{o}$, where $a$ is the lattice constant along the wire, and 
$a_{o}$ is the equilibrium value. Since $E / V=\frac{1}{2} C_{11} e_{11}^{2}$ by definition, we were able to fit our numerical results about the minimum to determine $C_{11}$ and $a_{o}$ for each wire. We found $C_{11}$ $=173,202,358 \mathrm{GPa}$, and $a_{o}=3.84,3.79,3.71 \AA$ for the 3 $\times 3,2 \times 2$, and $1 \times 1$ wires, respectively. These results should be compared with our bulk elastic constant calculated to be $C_{11}=102 \mathrm{GPa}$, and bulk lattice constant $a_{o}$ $=4.00 \AA$. Our numerical data clearly show that the smaller wires have a larger elastic constant, and a smaller equilibrium lattice constant along their length. This conclusion should be general and is not restricted to $\mathrm{Al}$ wires.

We can understand and organize these results using a very simple continuum model. Although quite approximate, this model turns out to work extremely well and it involves only two adjustable constants that we can fix using our first principles data. In fact there exists literature that demonstrated the usefulness of various harmonic methods for analyzing structural data. ${ }^{32,33}$ Here we construct a model that is specifically useful for analyzing the nanowire results obtained from the QMD simulations. Let us represent the surface bonds of the wires by effective springs with a force constant $k_{s}$ and equilibrium bond length $d_{s}$. Similarly, we represent all bulk bonds approximately by springs with a force constant $k_{b}$ and equilibrium length $d_{b}$. With this model, the nanowire is then viewed as a continuous block of medium, and the total energy per length $a_{o}$ along the block due to the longitudinal bonds is

$$
E=N_{s} \frac{k_{s}}{2}\left(d-d_{s}\right)^{2}+N_{b} \frac{k_{b}}{2}\left(d-d_{b}\right)^{2},
$$

where $N_{s}$ and $N_{b}$ are the number of longitudinal surface and bulk bonds per length $a_{o}$ along the wire. Within this continuum model the transverse bonds do not explicitly enter into our calculation of $C_{11}$ along the wire length. After some simple manipulation, the energy equation can be rewritten as $E=\frac{1}{2}\left(N_{s} k_{s}+N_{b} k_{b}\right)\left(d-d_{o}\right)^{2}$ up to an additive constant, where

$$
d_{o}=\frac{N_{s} k_{s} d_{s}+N_{b} k_{b} d_{b}}{N_{s} k_{s}+N_{b} k_{b}} .
$$

Now since $d=a / \sqrt{2}$ for our Al fcc nanowires, we can express the energy as $E=\frac{1}{2}\left(N_{s} k_{s}+N_{b} k_{b}\right)\left(a-a_{o}\right)^{2} / 2$. Dividing by the volume $V=\left(W a_{b}\right)^{2} a_{o}$ for a $\mathrm{W} \times \mathrm{W}$ wire, we conclude that

$$
E / V=\frac{1}{2} a_{o}\left[\frac{N_{s} k_{s}+N_{b} k_{b}}{2 a_{b}^{2} W^{2}}\right] e_{11}^{2},
$$

and by now comparing this expression with the definition of $C_{11}$, we deduce that

$$
C_{11}=\frac{a_{o}}{2 a_{b}^{2}}\left[\frac{N_{s} k_{s}+N_{b} k_{b}}{W^{2}}\right],
$$

where for a $\mathrm{W} \times \mathrm{W}$ nanowire, it can easily be shown that $N_{s}=16 \mathrm{~W}$ and $N_{b}=8 \mathrm{~W}(2 \mathrm{~W}-1)$.

The only unknowns in Eq. (4) are the force constants $k_{s}$ and $k_{b}$, which we can easily solve for using our total energy numerical data. In particular, the bulk data should give a

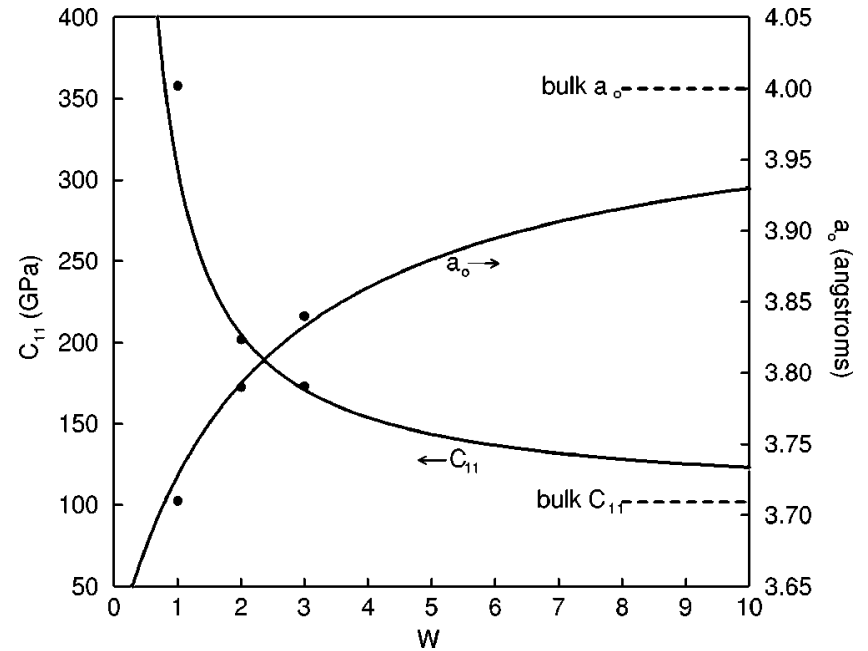

FIG. 3. Plots of $C_{11}$ and $a_{o}$ (along the wire length) for a $W$ $\times W$ nanowire, where the solid lines are the $C_{11}$ and $a_{o}$ given by our model, and the dots are the results from our first-principles calculation. The bulk elastic constant and bulk lattice constant are denoted by the dashed lines.

good estimate for $k_{b}$. For bulk, $W \rightarrow \infty$ and $a_{o}=a_{b}$ hence we conclude from Eq. (4) that $C_{11}=8 k_{b} / a_{b}$. Using our numerical data for bulk, $C_{11}=102 \mathrm{GPa}$ and $a_{b}=4.00 \AA$, we conclude that $k_{b}=0.32 \mathrm{eV} / \AA^{2}$. Next we solve for $k_{s}$ using the nanowire $C_{11}$ data; the value of $k_{s}$ can be found using just one wire, and it should be approximately independent of which $C_{11}$ is used. For example, using the $3 \times 3$ elastic constant data we conclude that $k_{s}=0.89 \mathrm{eV} / \AA^{2}$, and using the $2 \times 2$ data we get $k_{s}=0.86 \mathrm{eV} / \AA^{2}$. By taking an average of these results, we arrive at a final value $k_{s}=0.875 \mathrm{eV} / \AA^{2}$ for our continuum model, which implies that surface bonds are about three times stiffer than bulk bonds. Note that we have not used the $1 \times 1$ data in the $k_{s}$ average, because the interior bonds of the $1 \times 1$ wire do not exhibit perfect bulk characteristics due to its small size. By calculating the bond charges and comparing with the bulk, we found that the interior bonds of the $1 \times 1$ wire are stronger than bulk bonds, hence $k_{b}$ should actually be somewhat larger for this wire. Since we do not include this fact in our continuum model, the elastic constant of the $1 \times 1$ wire will not be accurately represented by the model. Replacing the $k_{b}$ and $k_{s}$ determined above into Eq. (4), the continuum model implies that $C_{11}$ $=171,205,307 \mathrm{GPa}$ for the $3 \times 3,2 \times 2$, and $1 \times 1$ wires. The model underestimates the elastic constant of the $1 \times 1$ wire by about $14 \%$, as should be expected from our above discussion. The results of the continuum model are summarized graphically in Fig. (3), where the first-principles data (dots) are compared to the model curve of $C_{11}$ given by Eq. (4). The solid curve thus gives the prediction of the elastic constant $C_{11}$ for $\mathrm{Al}$ atomic wires of various cross-sectional sizes. $C_{11}$ approaches the bulk value as $1 / W$, which simply reflects the contribution of surface energy to this mechanical property, as has been understood from continuum theory. ${ }^{7}$

Next, we evaluate the equilibrium bond lengths $d_{b}$ and $d_{s}$, using our $C_{11}$ data. We know from our bulk calculation that $d_{b}=a_{b} / \sqrt{2}=2.83 \AA$. We use this in conjunction with Eq. (2) and the nanowire data to solve for $d_{s}$. To be precise, we average the $d_{s}$ found using the $3 \times 3$ and $2 \times 2$ nanowire 
TABLE I. Average displacement due to relaxation, where the percentage is with respect to the bulk fcc bond length.

\begin{tabular}{lccc}
\hline \hline & Layer 0 & Layer 1 & Layer 2 \\
\hline Chain & $0.11 \AA$ & & \\
& $3.8 \%$ & & \\
$1 \times 1$ Wire & $0.08 \AA$ & $0.16 \AA$ & \\
No Corners & $2.9 \%$ & $5.9 \%$ & \\
$1 \times 1$ Wire & $0.07 \AA$ & $0.10 \AA$ & \\
& $2.4 \%$ & $3.6 \%$ & \\
$2 \times 2$ Wire & $0.07 \AA$ & $0.06 \AA$ & $0.10 \AA$ \\
& $2.5 \%$ & $2.2 \%$ & $3.7 \%$ \\
\hline \hline
\end{tabular}

data, from which we obtained $d_{s}=2.60 \AA$. To summarize these findings, we use the above results in conjunction with Eq. (2) and plot the continuum model prediction for the effective equilibrium "lattice" constant $a_{o}$ (along the wire length) in Fig. 3. Finally, note that since $d_{s}<d_{b}$, the surface atoms of an $\mathrm{Al}(100)$ surface must experience tensile stress. We can actually use the results of our continuum model to estimate the surface stress of an $\mathrm{Al}(100)$ surface. In particular, the surface stress can be expressed as ${ }^{34}$

$$
g_{x x}=\frac{N_{p} d_{b}}{2 A} \frac{k s}{2}\left(d_{b}-d_{s}\right),
$$

where $N_{p}$ is the number of nearest neighbors in the plane of a surface atom, and $A$ is the area per surface atom. In the case of $\operatorname{Al}(100) N_{p}=4$, and using our results for $d_{b}$ and $d_{s}$, we find that $g_{x x}=0.07 \mathrm{eV} / \AA^{2}$, which is comparable to the surface stress of Al found directly using a plane-wave firstprinciples calculation. ${ }^{34}$

\section{B. Dynamic}

We conducted numerous QMD simulations to determine the stability of the wires. In all the dynamical simulations, the end atoms of the wires were fixed so as to mimic pinning due to leads that attach to the wires in a device application (see Fig. 1). For the chain, we started with a bond length of of an infinite $\mathrm{Al}$ chain, $2.41 \AA$. For the thicker wires we started with the bulk atomic positions. At a temperature $T$ $=0 \mathrm{~K}, \mathrm{QMD}$ relaxes the wire structure to a local energy minimum. A summary of the average atomic displacement is presented in Table I. The average displacement for atoms in a given layer $i$ is defined as

$$
\overline{|\Delta \mathbf{R}|_{i}}=\frac{1}{N_{i}} \sum\left|\mathbf{R}_{r}-\mathbf{R}_{b}\right|
$$

where $N_{i}$ is the number of free atoms in layer $i, \mathbf{R}_{r}$ are the relaxed atomic positions, $\mathbf{R}_{b}$ are the bulk initial positions, and the sum is over the free atoms in layer $i$. Our results show that the relaxation for the larger wires, namely, the 1 $\times 1$ and $2 \times 2$ fcc wires are very similar. In particular, the outer layer atoms undergo a relaxation of about $3.5 \%$, whereas all the inner layers move by only $2.5 \%$.

Next we studied the influence of room temperature on the atomic positions. We conducted a QMD simulation at $300 \mathrm{~K}$ for 400 time steps for each of the wires, starting with the
TABLE II. Average displacement of each layer at $300 \mathrm{~K}$, where the percentage is with respect to the bulk fcc bond length.

\begin{tabular}{lccc}
\hline \hline & Layer 0 & Layer 1 & Layer 2 \\
\hline Chain & $0.47 \AA$ & & \\
& $16.6 \%$ & & \\
$1 \times 1$ Wire & $0.40 \AA$ & $0.39 \AA$ & \\
No Corners & $14.1 \%$ & $13.7 \%$ & \\
$1 \times 1$ Wire & $0.14 \AA$ & $0.22 \AA$ & \\
& $4.9 \%$ & $7.8 \%$ & \\
$2 \times 2$ Wire & $0.12 \AA$ & $0.12 \AA$ & $0.17 \AA$ \\
& $4.4 \%$ & $4.4 \%$ & $6.1 \%$ \\
\hline \hline
\end{tabular}

relaxed atomic coordinates. We then calculated the time average of the average displacement for atoms in each layer, which is defined as

$$
\left\langle\overline{|\Delta \mathbf{R}|_{i}}\right\rangle=\frac{1}{N_{\text {steps }}} \sum{\overline{|\Delta \mathbf{R}(t)|_{i}}}_{,}
$$

where $N_{\text {steps }}$ is the number of time steps used in the time average, $\overline{|\Delta \mathbf{R}(t)|}$ is the average displacement at time $t$ of atoms in layer $i$ with respect to the mean positions, and the sum is over the time steps. Using this definition, the average displacement of an atom in layer $i$ is found by starting the time average at the 25th time step, which insures that the system has had enough time to attain the desired temperature. The results of our calculations are summarized in Table II. As in the case of pure relaxation $(T=0)$, the outer atoms show more displacement, whereas the inner atoms displace by approximately $4-5 \%$ in the case of the $1 \times 1$ and $2 \times 2$ wires. On the other hand, the smaller wires exhibit much more atomic displacement; for example, the $1 \times 1$ wire with no corner atoms has an average displacement of $14 \%$, which is due to the fact that many of the bonds break, and the structure becomes unstable at the room temperature.

We can actually estimate the rms displacement of a bulk atom at room temperature using our continuum model. To accomplish this, we simply apply the equipartition theorem to a bulk atom and we get

$$
\sqrt{\left\langle|\Delta \mathbf{R}|^{2}\right\rangle}=\sqrt{\frac{3 k_{B} T}{N_{n} k_{b}}},
$$

where $k_{B}$ is Boltzmann's constant, $T$ is the temperature, $N_{n}$ is the number of nearest neighbors for a bulk atom, and $k_{b}$ is the force constant of bulk bonds. Next, by evaluating a few simple integrals, it can easily be shown that $\langle|\Delta \mathbf{R}|\rangle$ $=0.92 \sqrt{\left\langle|\Delta \mathbf{R}|^{2}\right\rangle}$. By using our result for $k_{b}$, we conclude from the above equation that at $300 \mathrm{~K}$ the average displacement of a bulk atom is $0.13 \AA$ or $4.6 \%$, which agrees quite reasonably with the average displacement of the inner nanowire atoms found with our QMD simulations (the layers 0 and 1 data for the $2 \times 2$ wire in Table II).

\section{TRANSPORT PROPERTIES}

With the atomic positions determined by QMD as discussed above, we now present the results of quantum conductance of these atomic wires in the form schematically 
shown in Fig. 1. In our calculation the long leads are modeled using the jellium model where the electrons are described by their wave functions while the ion charges are uniformly distributed inside the leads' volume. Using the QMD positions of the atoms, the transport properties are obtained in two steps: the evaluation of the self-consistent effective potential and the calculation of the quantum conductance.

In the first step of the conductance calculation, fixing the atomic positions as determined by the QMD, we have determined the self-consistent electronic potential $V_{\text {eff }}(\mathbf{r})$ $\equiv \delta U / \delta \rho(\mathbf{r})$, which is seen by all the electrons including those provided by the jellium leads. Here $U[\rho]$ is the total self-consistent potential energy while $\rho$ is the electron density. The particular reason for this procedure is to overcome the approximations related to the nature of the Harris ${ }^{24}$ functional used in our QMD method, and to introduce the leads to the atomic wire. Although there could be very slight differences concerning mechanical structures using the QMD of Sankey and Niklewski ${ }^{23}$ in comparison to the self-consistent plane-wave-based methods, ${ }^{35}$ these small differences are not important for our purposes here, thus we used the atomic positions of our QMD to determine $V_{e f f}$. In previous calculations involving only up to six atoms in the atomic section, ${ }^{21,22}$ we have determined $V_{\text {eff }}$ by solving the KohnSham self-consistent equation for the electronic wave functions using a plane-wave basis. For the large systems considered here, this step is completed by applying the ThomasFermi-von Weizsäcker (TFvW) density expanded kineticenergy functional, which is the subject of many recent investigations. ${ }^{36-39}$ For small wires with up to four atoms, we have checked that the TFvW approach and the planewave approach give consistent results for the dc conductance, but for the large wires studied here TFvW makes the investigations numerically less intensive. Since we consider cases in which each lead is asymptotically a perfect wire, we made sure that the length of the leads is long enough to capture the infinite length limit. This way the eigenstates obtained from the equilibrium density-functional calculation can be brought into the form of scattering states of the wire. $^{40}$

To directly obtain the scattering states, in the second step of the conductance calculation we solve a 3D quantum scattering problem of a particle traversing the atomic wire defined by $V_{e f f}$. For this purpose we have developed a 3D quantum scattering algorithm based on a transfer matrix evaluation of the scattering matrix. ${ }^{41,21}$ As a result we obtain transmission coefficients $T_{\alpha}(E)$, where the subscript $\alpha$ labels the transmission subbands. Finally the conductance of the wire is obtained by integrating $T_{\alpha}(E)$ together with the Fermi-Dirac distribution function in the standard fashion.

We concentrated on two atomic structures as the initial conditions of the QMD simulations, shown in Figs. 2(b) and 2(c). Figure 2(c) is a "thick" wire, with alternating stacks of four and five atoms in a fcc (100) arrangement ending with the five-atom stack at the ends. With nine stacks a thick wire has 41 atoms in the atomic section. The second initial condition, Fig. 2(b), is created by only keeping the atoms at the faces of the fcc bulk structure, thus the stacks are alternating with four and one atoms and the wires end with the fouratom stack. We call this wire a "thin" one. With fifteen

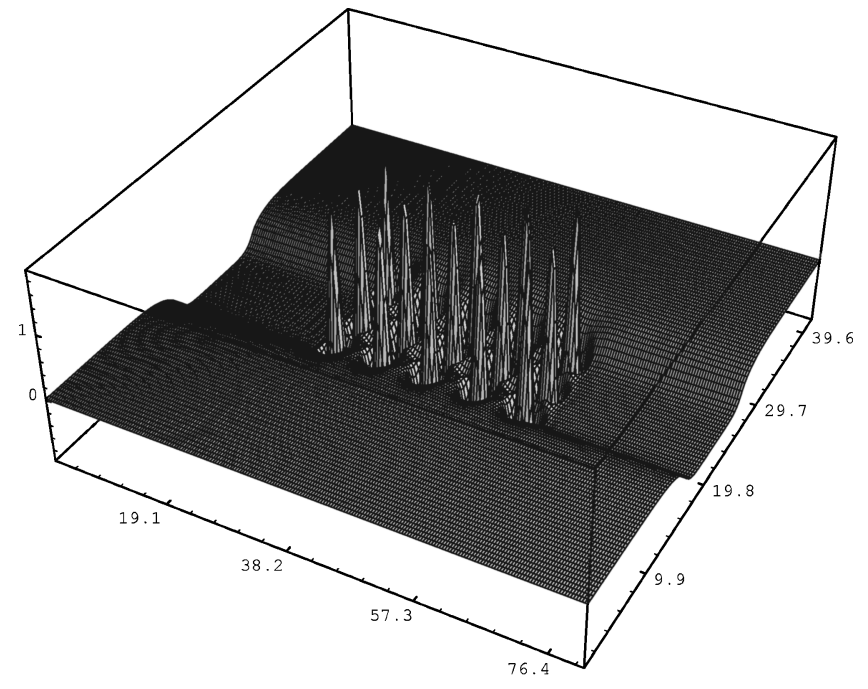

FIG. 4. The ground-state effective potential $V_{e f f}$ along the (110) direction obtained from the $a b$ initio total-energy calculation for a wire with 41 atoms relaxed at $0 \mathrm{~K}$. The scales are in a.u. units.

stacks the thin wire has 39 atoms in the atomic section. The QMD simulations of these systems were reported in the last section. For both the thick and thin wires at $0 \mathrm{~K}$, the final configuration has reached the fully relaxed state, which is somewhat different from the bulk structure. On the other hand, at $300 \mathrm{~K}$ the atomic positions in both cases are quite disordered (see last section). The distorted atomic configurations of the wires provide a natural positional disorder to the quantum conduction.

We have used the bulk $\mathrm{Al}$ electron density $r_{s}=2.07$ a.u. in the jellium leads. A lead has a cross-section of 10.52

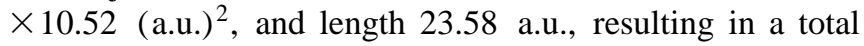
of 129 electrons in the leads. The whole system, leads plus the atomic section, is put into a supercell (see Fig. 1) of size $41.68 \times 41.68 \times 81.33$ (a.u.) $)^{3}$ for the "thick" wire case, and $37.24 \times 37.24 \times 104.01$ (a.u.) ${ }^{3}$ for the "thin" wire case, for standard density-functional analysis which produces a selfconsistent effective potential $V_{\text {eff }}(\mathbf{r})$ as seen by all the electrons. We have used an energy cutoff of $32 \mathrm{Ry}$. Figure 4 shows $V_{\text {eff }}$ for a "thick" wire relaxed at $0 \mathrm{~K}$. The potential $V_{e f f}$ in the 3D leads is affected by the atoms near the leadatom junction, but this effect is damped out away from the junction indicating that the length of the leads is long enough to give a good approximation of the infinite length limit. In calculating $V_{e f f}$ the numerical convergence is guaranteed within a few $\mu \mathrm{eV}$. In the leads, $V_{e f f}$ is essentially a well with a depth $\sim-0.51$ a.u. below the Fermi level in the crosssection plane. The bonding between the $\mathrm{Al}$ atoms, and between atoms and the jellium leads, is clearly obtained. In the lateral direction, far away from the atomic section, a higher potential is seen that reflects the vacuum. In the atomic section, $V_{\text {eff }}$ has rather high peaks due to the ionic core repulsion of the atoms, and surrounding the peaks there is the usual attractive part of atomic potential.

Figure 5 shows the conductance $G(E)$ as a function of incoming electron energy $E$ for the "thick" wires. The three curves are for wires relaxed at $0,300 \mathrm{~K}$ and no dynamic relaxation. In all cases some degree of conductance "plateau" is observed. ${ }^{42}$ However the quantization of $G(E)$ is 


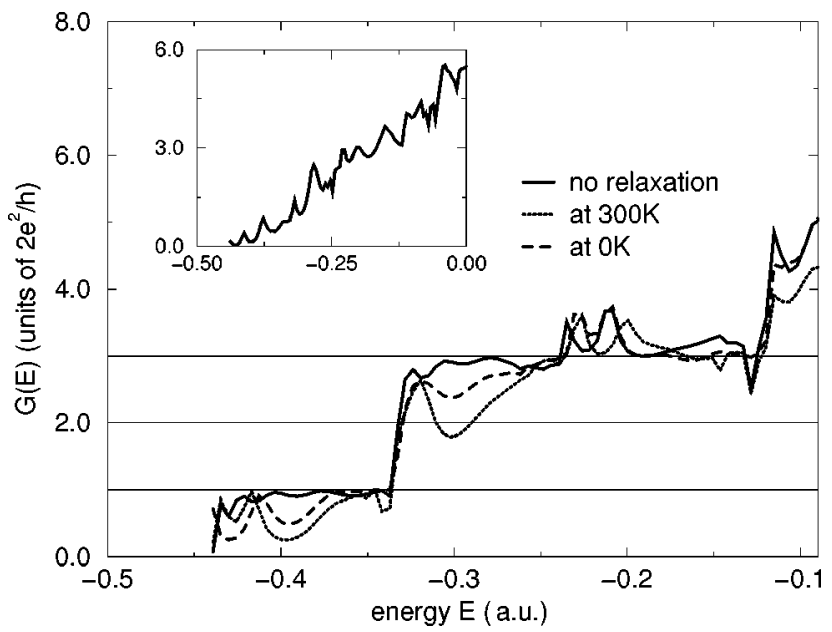

FIG. 5. Conductance $G(E)$ as a function of incoming electron energy $E$ for wires in the thick configuration (41 atoms) with fixed ends. The calculated Fermi level $E_{f}$ is about -0.106 a.u. Inset: $G(E)$ for a thick wire relaxed at $0 \mathrm{~K}$ with free ends.

not perfect, reflecting the fact that the dynamically relaxed atomic wires have some degree of positional disorder. For the wire without relaxation, the quantization is better due to the crystalline structure of the atomic section. However the atom-lead junction still provides scattering to the electrons which gives rise to quantum mode mixing, leading to the imperfections of the quantization and the resonance behavior. In addition there is a resonance behavior in between energies -0.2 to -0.25 a.u. for all three cases as $G(E)$ shows two peaks there. This is also likely to be due to the scattering at the atom-lead junction. Figure 5 also shows the relative importance of the positional disorder as a result of the dynamic relaxation: clearly the $300 \mathrm{~K}$ curve shows the worst quantization. Our analysis included two effects of temperature, one being the influence on the atomic positions, the other is the smearing effect of the transmission coefficient when we compute $G(E)$. We emphasize that the smearing effect has little influence on $G$ because of the very large energy scales involved in the atomic wires in comparison to the temperature. Precisely for this reason, a quantized $G$ can survive elevated temperatures as demonstrated experimentally ${ }^{6,13,14}$ and shown here. For the large wires studied here, the ideal quantization, namely, the quasi-1D behavior, cannot be precisely established due to various scattering and mode mixing. This is quite different from cases where only a chain of a few atoms are included in the atomic section. In that case very good conductance quantization can be obtained. Indeed, the experimentally fabricated atomic wires rarely showed perfect quantization ${ }^{1,15,6}$ presumably due in part to the same reason as we found here. The inset of Fig. 5 plots $G(E)$ for a thick wire, dynamically relaxed at 0 $\mathrm{K}$ for $1.8 \mathrm{ps}$ with the ends of the atomic section free to move instead of being fixed. Our QMD simulation showed that in this wire the atomic positions are completely disordered. $G(E)$ clearly reflects this fact and shows no quantization at all.

The results for the thin configurations display quite interesting features, as plotted in Fig. 6. This exercise clearly shows that the structure of an atomic wire can have drastic influence on the transport properties. As shown by the

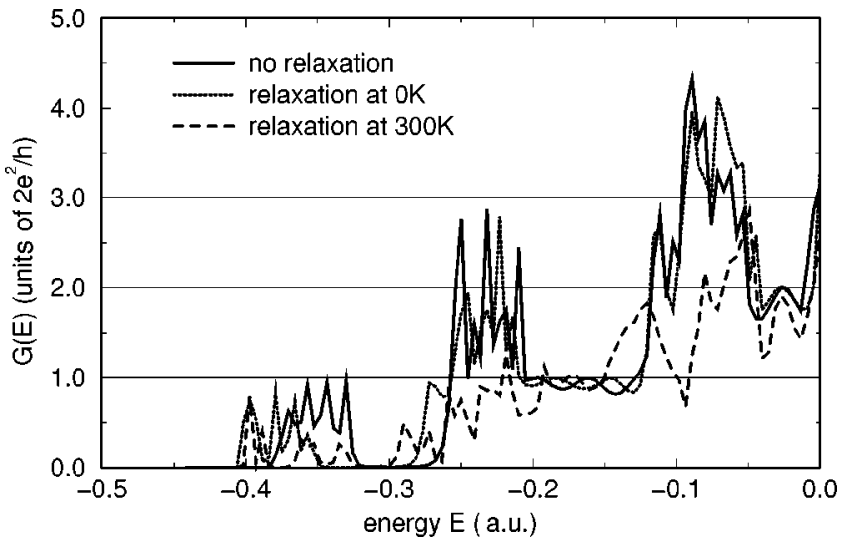

FIG. 6. Conductance $G(E)$ for wires in the thin configuration (39 atoms) with fixed ends. The calculated Fermi energy is about $E_{f}=-0.108$ a.u. There are two "gaps" in $G(E)$.

curves, rather than being quantized, $G(E)$ for the thin wires seems to show a transport "band gap," at energies around -0.3 a.u. and a "plateau" at -0.16 a.u. below the Fermi energy. The width of these regions are slightly narrower for the wire without dynamic relaxation. This seemingly strange result can be confirmed by investigating the DOS of the atomic section, which we obtained in our QMD simulations. Indeed, below the Fermi energy there is a band gap and a plateau in the DOS in the similar ranges of energies. This consistency is expected since linear response theory demands that the conductance is proportional to DOS. In addition, we have verified that the behavior of $G(E)$ for the thick wires is also consistent with that of the DOS where no band gaps were obtained.

\section{DISCUSSION AND SUMMARY}

This work focuses on two important aspects of atomic scale wires, the structural property and the related quantum conduction. Our calculations are from first principles by combining the quantum molecular dynamics, the evaluation of the self-consistent effective potential, and the solution of a 3D quantum scattering problem. This is a powerful approach to investigate systems where atomic degrees of freedom are important.

On the structural simulations, we have conducted both static and dynamic first principles calculations to determine some mechanic properties of aluminum nanowires of various thickness. The elastic constant along the wire length was found to be larger for wires with a smaller thickness, which can be explained by the fact that surface bonds are much stronger than bulk bonds. In other words, at the atomic scale, the surface energy plays an important role. Our QMD simulations show that in the case of the most stable wire, relaxation causes about a $2-4 \%$ change in atomic position, whereas room temperature contributes another 4-6\%. We thus conclude that atomic disorder due to relaxation and room temperature give rise to a net atomic displacement of $6-10 \%$ for the atomic wires studied here, where the wire ends are pinned to mimic the existence of leads. Such a positional disorder in turn alters the electrical transport properties such as the conductance quantization. Clearly, for larger wires the average atomic displacement becomes 
smaller. So far studies of structures usually assumed a classical dynamics for the ions. At low temperature the quantum zero-point motion and quantum dynamics of the ions can play a role and even change the conclusion one might draw such as which structure gives the global energy minimum. These effects can be important to distinguish structures with energies very close to each other at low temperatures. The above simulations were for free-standing wires, which can be fabricated and studied experimentally by extracting a STM tip from a surface. ${ }^{6}$ For wires microfabricated on top of substrates, ${ }^{1}$ our results place upper limits on the displacement of atoms. It is interesting to see that the very simple continuum model, with only two parameters, can partially account for the simulation data. The model is quite intuitive in giving us some general understanding of the structural properties of atomic wires. Through the two constants, which we obtain from our ab initio data, the results can be organized in a very clean fashion, which allows for much more general predictions. We thus expect this and similar models to be useful in conjunction with ab initio modeling for further investigations of various nanosystems.

We found that positional disorder and atomic arrangements can have a very important effect on the conductance at this atomic level, hence for potential quantum device applications of the atomic wires, one must carefully control the quality of the atomic structure in order to obtain good conductance quantization. There are several possible further extensions of the present work in the investigations of quantum transport through atomic systems. First, the atomic wires studied here is several times smaller than those fabricated in the labs. ${ }^{1,5}$ In order to approach the experimental sizes, verylarge-scale calculations are needed and there is a need to refine the numerical procedure. Second, it will be useful to more carefully correlate the structural information with conductance behavior. To this purpose many temperature annealings of the structures are desired from the QMD simulations. Again, this requires very large computation effort. Third, in this work we have not considered the explicit dephasing effect on quantum waves in the scattering process. This will be an important issue for further analysis. Fourth, the details of our density-functional-theory analysis can be extended using generalized gradient approximation and other kinetic energy functionals. ${ }^{43}$ These improvements can be valuable for more complicated systems. Finally, our analysis can be extended to multilead systems and this will make $a b$ initio simulations of atomic quantum functional devices possible.

\section{ACKNOWLEDGMENTS}

H.G. thanks Professor C.T. Chan of The University of Science and Technology of Hong Kong for a useful conversation concerning the elastic properties of nanowires. We gratefully acknowledge support from NSERC of Canada and FCAR of Québec. J.W. acknowledges support by the SAR Government of Hong Kong under Grant No. HKU 261/95P, and the Croucher Foundation. We also thank the Cornell Theory Center and the Computer Center of the University of Hong Kong for substantial CPU allocations.
${ }^{1}$ H. Namatsu, Y. Takahashi, M. Nagase, and K. Murase, J. Vac. Sci. Technol. B 13, 2166 (1995).

${ }^{2}$ D. Routkevitch, T. Bigioni, M. Moskovits, and J. M. Xu, J. Phys. Chem. 100, 14037 (1996).

${ }^{3}$ H. I. Liu et al., Appl. Phys. Lett. 64, 1383 (1994).

${ }^{4}$ A. A. Setlur, J. M. Lauerhaas, J. Y. Dai, and R. P. H. Chang, Appl. Phys. Lett. 69, 345 (1996).

${ }^{5}$ Yasuo Wada et al., J. Vac. Sci. Technol. B 12, 48 (1994).

${ }^{6}$ J. I. Pascual et al., Science 267, 1793 (1995); Phys. Rev. Lett. 71, 1852 (1993); J. Vac. Sci. Technol. B 13, 1280 (1995).

${ }^{7}$ See, for example, E. Rabinowicz, Friction and Wear of Materials (Wiley, New York, 1995).

${ }^{8}$ Ali Yazdani, D. M. Eigler, and N. D. Lang, Science 272, 1921 (1996); N. D. Lang, Phys. Rev. B 52, 5335 (1995).

${ }^{9}$ J. K. Gimzewski and R. Möller, Phys. Rev. B 36, 1284 (1987).

${ }^{10}$ U. Dürig, O. Züger, and D. W. Pohl, Phys. Rev. Lett. 65, 349 (1990).

${ }^{11}$ N. Agrait, J. G. Rodrigo, and S. Vieira, Phys. Rev. B 47, 12345 (1993).

${ }^{12}$ J. M. Krans et al., Phys. Rev. B 48, 14721 (1993).

${ }^{13}$ L. Kuipers and J. W. M. Frenken, Phys. Rev. Lett. 70, 3907 (1993).

${ }^{14}$ M. Brandbyge et al., Phys. Rev. B 52, 8499 (1995); L. Olesen et al., Phys. Rev. Lett. 72, 2251 (1994).

${ }^{15}$ E. S. Snow, D. Park, and P. M. Campbell, Appl. Phys. Lett. 69, 269 (1996).

${ }^{16}$ J. A. Torres and J. J. Sáenz, Phys. Rev. Lett. 77, 2245 (1996); A.
Garcia-Martin, J. A. Torres, and J. J. Sénz, Phys. Rev. B 54, 13 448 (1996).

${ }^{17}$ R. N. Todorov and A. P. Sutton, Phys. Rev. Lett. 70, 2138 (1993).

${ }^{18}$ M. P. Samanta et al., Phys. Rev. B 53, R7626 (1996); S. Datta and W. Tian, ibid. 55, R1914 (1997).

${ }^{19}$ Eldon Emberly and George Kirczenow, cond-mat/9807290 (unpublished).

${ }^{20}$ L. Chico, L. X. Benedict, S. G. Louie, and M. L. Cohen, Phys. Rev. B 54, 2600 (1996).

${ }^{21}$ C. C. Wan et al., Appl. Phys. Lett. 71, 419 (1997).

${ }^{22}$ J. L. Mozos et al., Phys. Rev. B 56, R4351 (1997).

${ }^{23}$ O. F. Sankey and D. J. Niklewski, Phys. Rev. B 40, 3979 (1989).

${ }^{24}$ J. Harris, Phys. Rev. B 31, 1770 (1985).

${ }^{25}$ D. R. Hamann, M. Schlüter, and C. Chiang, Phys. Rev. Lett. 43, 1494 (1982).

${ }^{26}$ O. F. Sankey, D. J. Niklewski, D. A. Drabold, and J.D. Dow, Phys. Rev. B 41, 12750 (1990).

${ }^{27}$ S. H. Yang, D. A. Drabold, J. B. Adams, and A. Sachdev, Phys. Rev. B 47, 1567 (1993).

${ }^{28}$ G. B. Adams and O. F. Sankey, J. Vac. Sci. Technol. A 10, 2046 (1992).

${ }^{29}$ D. A. Drabold, P. A. Fedders, O. F. Sankey, and J. D. Dow, Phys. Rev. B 42, 5135 (1990).

${ }^{30}$ A. Caro, D. A. Drabold, and O. F. Sankey, Phys. Rev. B 49, 6647 (1994).

${ }^{31}$ R. Biswas and D. R. Hamann, Phys. Rev. B 34, 895 (1986).

${ }^{32}$ S. M. Foiles, Phys. Rev. B 49, 14930 (1994). 
${ }^{33}$ A. P. Sutton, Philos. Trans. R. Soc. London, Ser. A 341, 233 (1992).

${ }^{34}$ R. J. Needs and M. J. Godfrey, Phys. Rev. B 42, 10933 (1990). We have divided Needs' result by 2 since his force constant is actually half of ours.

${ }^{35}$ For a review, see, for example, M.C. Payne et al., Rev. Mod. Phys. 64, 1045 (1992).

${ }^{36}$ N. Govind, José-Luis Mozos, and Hong Guo, Phys. Rev. B 51, 7101 (1995); N. Govind, Jian Wang, and Hong Guo, ibid. 50, 11 175 (1994).

${ }^{37}$ M. Pearson, E. Smargiassi, and P. A. Madden, J. Phys.: Condens. Matter 5, 3221 (1993).
${ }^{38}$ L. W. Wang and M. P. Teter, Phys. Rev. B 45, 13196 (1992).

${ }^{39}$ E. Chacón, J. E. Alvarellos, and P. Tarazona, Phys. Rev. B 32, 7868 (1985).

${ }^{40}$ M. Büttiker, in Quantum Dynamics of Submicron Structures, edited by H.A. Cerdeira et al. (Academic, Amsterdam, 1995).

${ }^{41}$ Wei-Dong Sheng and Jian-Bai Xia, Phys. Lett. A 220, 268 (1996).

${ }^{42}$ Notice that the "plateau" at $2 \times 2 e^{2} / h$ is missing. For a $3 \mathrm{D}$ wire with a square cross section, the transverse quantization gives conductance steps of $1,3,4,6, \ldots$ in units of $2 e^{2} / h$.

${ }^{43}$ A. Lembarki and H. Chermette, Phys. Rev. A 50, 5328 (1994); P. Fuentealba and O. Ryes, Chem. Phys. Lett. 232, 31 (1995). 